\title{
Are changes in performance with noise and age due to adrenergic arousal?
}

\author{
J. RICHARD JENNINGS, RICHARD STILLER, and KAY BROCK \\ Western Psychiatric Institute and Clinic, University of Pittsburgh, Pittsburgh, Pennsylvania
}

\begin{abstract}
The associations among plasma catecholamine levels, cardiovascular change, and performance efficiency were examined in young and old men $(N=26)$ who performed a laboratory task while exposed to noise. The aim of the experiment was to determine whether performance differences related to noise and aging could be explained by neuroendocrine arousal. According to inverted$\mathrm{U}$ arousal theories, overarousal due to noise or aging should narrow the focus of attention, altering performance. Older men were expected to show high basal catecholamine levels, which were expected to accentuate the deficit due to noise predicted by inverted-U arousal theories. Performance changes, cardiovascular changes, and age-related increases in catecholamines were observed. Performance was not, however, related to these changes or to catecholamine levels (which did not change significantly over the course of the task). Individual differences in task-induced changes in epinephrine did, however, show an inverted-U relationship with mean reaction speed. Brief, task-induced electrophysiological responses suggested an alternative view, that aging and noise induce specific rather than general changes in performance and autonomic/neuroendocrine support.
\end{abstract}

Research on arousal and aging has been reviewed by Woodruff (1985) and Marsh and Thompson (1977). Some evidence suggests that the elderly are underaroused and other evidence that they are overaroused. Typically, the evidence for underarousal shows that task-induced responses of an electrophysiological measure, such as skin conductance, are smaller in the old than in the young ( $\mathrm{Co}-$ hen \& Shmavonian, 1967). The evidence for overarousal in the old relative to the young typically shows a heightened endocrine response to a task and poor performance (Eisdorfer, 1968).

Conceptually conflicting results such as these have led to questions about the validity of the concept of general arousal. Increasingly, the specificity rather than the generality of neural control has been emphasized (e.g., Jennings, 1986; Lacey, 1967). Taken together, these difficulties in relating aging and arousal led us to critically reexamine prior work in which performance changes had been attributed to arousal. Typically, a stressor, such as noise, is assumed to induce arousal, and no concurrent examinination of such measures of arousal as performance, physiology, or self-report is made. When we performed an experiment that included such measures (Jennings, Brock, \& Nebes, 1988b), we found little evidence for the concept of general arousal. Our volunteers performed two concurrent tasks in 90-dBA white noise or relative quiet. Noise altered their performance and induced self-reports of mild stress, but failed to alter cardiovascular responses consistently. Before task performance, however, the older volunteers reported themselves (on the

The authors gratefully acknowledge the support of NIA Grant AG03792 and wish to thank Thomas Kamarck for his critical reading of the manuscript. Reprint requests should be sent to J. R. Jennings, 3811 O'Hara St., WPIC 13th floor, Pittsburgh, PA 15213.
Stress-Arousal Check List, SACL; Mackay, 1980) as being more aroused than did the younger volunteers, and they were found to have higher heart rate, blood pressure, and pulse-wave velocity (PWV) than the younger volunteers.

The purpose of the present experiment was to compare catecholamine levels and reactivity with cardiovascular levels and reactivity in the same dual-task/noise paradigm that had been employed previously. To do this, we tested two specifications of arousal theory: in one, older individuals show arousal levels that are not optimal for subsequent performance; in the other, individuals give arousal responses to a stressor that are not optimal for concurrent performance. Much of the evidence for overarousal in the elderly comes from studies of catecholamine levels and reactivity (Woodruff, 1985). Due to the relatively brief time course of neural action, the arousal state is perhaps better represented in endocrine levels than in measurements of the state of the autonomic and central nervous system. If so, then catecholamine levels and reactivity to noise might be expected to be higher in older than in younger volunteers, and individual differences in performance would be related to individual differences in catecholamines. Norepinephrine levels are generally thought to be elevated in the elderly (e.g., Wilkie et al., 1985; Ziegler, Lake, \& Kopin, 1976) and epinephrine may also be elevated (Fleg, Tzankoff, \& Lakatta, 1985). Age, however, has not been shown to be a simple determinant of reactivity of the catecholamines to physical and psychological stressors (Fleg, Tzankoff, \& Lakatta, 1985; Goldberg, Kreider, \& Roberts, 1984; Sachs, Hamberger, \& Kaijser, 1985).

The present study examined arousal and performance across age by assessing heart rate, central and peripheral 
vascular function, dopamine, epinephrine, norepinephrine, and self-reports of stress arousal in a dual task performed in 90-dBA continuous white noise. An integer addition task was combined with a reaction-time (RT) task for visual detection. Stimuli for the RT task were varied in probability in order to show the expected effect of noise on performance-a decrease in the efficiency of responding to low-likelihood-low-salience aspects of a complex task (eg., Hockey, 1973, 1983). At one point in the task, one of two sets of RT stimuli (light-emitting diode, LED, pairs) was likely to occur and one set might be more likely to occur than the other. At another point, RT stimuli could occur, but the probability of occurrence was low, and again one of the two RT stimuli could be particularly unlikely. If noise induces an overarousal, then performance should decrease for the low-probability lights relative to both high-probability lights and performance in quiet (Easterbrook, 1959). Young volunteers showed this effect in Jennings et al. (1988b). If old volunteers were to become overaroused, their performance ought to decline even more. In fact, however, the older volunteers failed to show this effect, thereby putting mediation by arousal into question. We sought to replicate this performance-age interaction and to examine its relation to catecholaminedefined arousal.

\section{METHOD}

\section{Volunteers}

To minimize reactions to novelty, 26 male volunteers were drawn from among subjects who had participated in two related studies. The 13 college volunteers were between 18 and 25 years of age; the 13 other volunteers were between 65 and 75 years of age and were selected from a registry of community-resident elderly volunteers. All of the volunteers were screened for health problems, drug use, hearing loss, and educational level (a minimum of one semester of college credits). Any volunteers who, in the audiology examination, were found to have hearing losses other than specific highfrequency losses (no greater than $25 \mathrm{~dB}$ loss at frequencies above $4000 \mathrm{~Hz}$ ) were excluded.

\section{Experimental Task}

The volunteers were asked to perform two concurrent tasks. The primary task was to add four integers presented individualy at 7sec intervals. The integers, varying between 0 and 9 , were selected randomly. The secondary task measured the time the volunteers required to detect the illumination of one of two pairs of LEDs and to press a microswitch response key corresponding to the pair, as quickly as possible.

The display board, a $66 \times 66 \mathrm{~cm}$ Plexiglas sheet painted a flat black color was mounted on a microphone stand that was adjustable so that the integer display could be at eye level for the semireclining volunteer. A $3 \times 3 \mathrm{~cm}$ seven-segment LED display at the center of the board provided the integer presentation. The peripheral lights were four LEDs, placed at the corners of the display board. The LEDs were illuminated in diagonal pairs-that is, top left and bottom right, top right and bottom left-in order to avoid positional biases on the part of the volunteers. The panel was placed so that the LEDs were at a visual angle of $25^{\circ}$ relative to the central integer. The illumination of the pair lasted $50 \mathrm{msec}$. The diagonal lights were illuminated $.5 \mathrm{sec}$ before or after integers were presented. The probability that an LED array would accompany an integer was .5. If an LED was to accompany an integer, the probability of its occurring before the integer was .7; after the integer, it was .3. Microswitch response keys appropriate for the handedness of the volunteer were placed on the arm of the reclining chair.

A sequence of four integers to be summed was termed a trial. The initiation of a new trial was signaled by a .5 -sec flash of all four corner LEDs. The first integer occurred $7 \mathrm{sec}$ later, followed at 7-sec intervals by the other three. A feedback display followed the last integer presentation by $3 \mathrm{sec}$ and remained illuminated for $1 \mathrm{sec}$. It also served as the signal to verbalize the sum. The volunteers were told that the arithmetic task was primary, and they were paid 4 cents for each correct sum and 2 cents for each correct-answer RT that was faster than $.5 \mathrm{sec}$. The feedback display illuminated all four corner LEDs, and the central display indicated the number of correct and aceptably fast RTs for that integer trial. RTs were measured in milliseconds using the clock on the DEC MINC computer. The accuracy of the choice response (expressed as proportion correct, $\mathrm{PC}$ ) was scored simply by computing the proportion of trials in which the response key signaled by the LED array was pressed.

Noise was produced by a Lafayette Instruments white-noise generator and was presented over Beltone headphones. These were calibrated weekly with a GenRad sound-level meter and an earphone coupler. The GenRad sound-level meter was calibrated on an A weighting with a MINICAL $1-\mathrm{kHz}$ sound-level calibrator.

\section{Procedure}

The volunteers participated in two sessions on separate days, with Session 1 lasting $1 \mathrm{~h}$ and Session 2 lasting $2.5 \mathrm{~h}$. In Session 1, they practiced the RT and arithmetic tasks until they had demonstrated a capability to achieve incentive pay for their performance. Session 2 was divided into two performance-task conditions: a 30-trial set of the performance task with an equal probability that the presented diagonal LEDs would appear on the right or left side, and a 30-trial set with a bias to either the right or the left diagonal. The volunteers were informed of the biasing. The side of the bias and the order of conditions for each subject were determined by flipping a coin. The experimental session began with the attachment of the band electrodes that measured impedance. The volunteer then assumed a semireclining position for the remainder of the session. An intravenous catheter was inserted in the nondominant arm by a trained medical technician. This procedure was followed by application of the remaining physiological equipment. A $10-\mathrm{min}$ baseline period preceded the two 30-trial sets of performance tasks. During the baseline period, the display board was unplugged and the physiological signals were recorded while the volunteer listened to 50-dBA white noise. After the baseline period, one 5-ml blood sample was drawn. At least 20 min elapsed between catheterization and collection of the blood sample. During the performancetask sets, the volunteers were exposed to $90 \mathrm{dBA}$ of white noise. Between the two sets, a second 5-ml blood sample was drawn and the SACL was employed. After the second performance-task set, the final 5-ml blood sample was drawn, and the volunteer then completed a postexperimental questionnaire which assessed his perception of the noise and indicated any task strategies he had developed to maintain or improve his performance.

\section{Physiological and Endocrine Measures}

Electrophysiological measures were designed to assess vagal changes (beat-by-beat heart rate), beta sympathetic changes (shortening of preejection period, increased systolic pressure), and alpha sympathetic changes (shortened peripheral vascular transit time and higher pulse-wave velocity). Heart rate was measured as interbeat intervals (IBIs) between the $R$ waves on a modified Lead 3 electrocardiogram, using the computer clock with an accuracy of $1 \mathrm{msec}$. Ten heartbeats were recorded for each integer presentation: five beats before the integer, one beat for the integer, and four beats after the integer. 
A Minnesota cardiogmph, Model 304B, was used to assess thoracic vascular impedance. A four-impedance-band system was used. The first impedance band was placed around the base of the neck, with the second band 2 to $3 \mathrm{~cm}$ above the first. The third impedance band was placed around the xiphoid process, and the fourth was positioned 3 to $4 \mathrm{~cm}$ below the third. Beckman electrode electrolyte was applied to the electrode tape and two electrocardiogram electrodes to insure complete skin contact.

Peripheral vascular activity was monitored with the pulse obtained from a photo plethysmograph attached to the thumbnail of the volunteer's nondominant hand. A computer program converted the signals from the photo and impedance devices into pulse-onset times and wave amplitude. The program identified the peak of each wave and also the maximum systolic slope. This time point was defined as pulse onset. The maximum slope, slope to peak amplitude, and onset time were used as measures, although the amplinde and slope were so highly correlated that only the latter will be presented. The onset times were expressed as pulse transit times (PTTs) from the $R$ wave of the electrocardiogram for each site. A PWV was computed by dividing the distance between the pulse-wave sites by the difference of the onset times for the impedance and thumb-pulse waves. These measures were collected on a beat-by-beat basis for the same beats collected for the IBI measure. The details of the photo plethysmograph used, the techniques, and the measures may be found in Jennings, Tahmoush, and Redmond (1980) and in Jennings and Choi (1983b).

Blood pressure was measured using a surface transducer, placed next to a standard arm cuff, and preamplifier from Grass Instruments. Cuff pressure and Korotkoff sound detections were recorded on the polygrpah. Pressures were taken at 5 -min intervals during each of the performance-task sets and the baseline period.

Heart sounds were monitored using an Instrumentation for Medicine chest microphone placed over the heart in conjunction with the Minnesota impedance cardiograph machine. The systolic time intervals were derived by combining information from heart sounds, impedance signals, and the electrocardiogram. The signals from five consecutive trial blocks were averaged, the times were hand read from averaged signals, and the intervals were calculated using the computer.

Respiration was measured using a Yellow Springs thermistor placed at the opening of the left nostril. This measure was used only to insure that no extreme respiratory maneuvers occurred that would create artifactual changes in the cardiovascular measures.

Catecholamines were measured in plasma. A special BectonDickinson Vacutainer, supplied by Upjohn Diagnostics (Kalamazoo, MI) and containing ethylene glycol-bis (B-aminoethyl ether) N, N-tetraacetic acid (EGTA), and glutathione, was used. The procedure required an intracatheter infusion set placed in the anticubital vein, flushed with a heparin solution $(10 \mathrm{U} / \mathrm{ml})$ and occluded (heparin lock). Since catecholamines tend to rise in a stimulated volunteer, the baseline sample was drawn at least 20 min after catheterization, and the first $0.5 \mathrm{ml}$ of blood was discarded. To avoid hemolysis, the blood tube was mixed by inversion without shaking and placed in an ice bath. The vacutainer tube was then immediately centrifuged in a refrigerated centrifuge. The plasma was immediately removed through a disposable pipet into a screw-capped polypropylene container and stored at below $-20^{\circ} \mathrm{C}$. The samples had to be clear before analysis.

The catecholamine analysis procedure was a single isotope assay modified from Passon and Peuler (1973). It was carried out with a catecholamines radioenzymatic assay kit $\left({ }^{3} \mathrm{H}\right)$, Cat-A-Kit, supplied by Upjohn Diagnostics. This assay measured norepinephrine, epinephrine, and dopamine in less than $1 \mathrm{ml}$ of plasma to a sensitivity of less than $20 \mathrm{pg}$ for dopamine and $5 \mathrm{pg}$ for norepinephrine and epinephrine. This procedure employed the enzyme catechol-O-methyl-transferase to transfer an ${ }^{3} \mathrm{H}$-methyl group from S-adenosyl-L-methione ( ${ }^{3} \mathrm{H}$-methyl) to form ${ }^{3} \mathrm{H}$ normetanephrine, ${ }^{3} \mathrm{H}$-metanephrine, and ${ }^{3} \mathrm{H}-3$-methoxytryamine.
These products were isolated by thin-layer chromatography (TLC). oxidized to ${ }^{3} \mathrm{H}$-vanillin with periodate, and extracted from the TLC plate. The individual catecholamines were determined by scintillation counting. Intra-assay variability relative to known standards was $4.3 \%$ for norepinephrine, $3.7 \%$ for epinephrine, and $4.7 \%$ for dopamine; comparable inter-assay figures were $7.6 \%, 10.7 \%$, and $12.7 \%$.

\section{Data Analysis}

The overall statistical approach was a repeated measures analysis of variance with a between-subjects factor of age group (Winer, 1971). LED probability bias and timing of LEDs before and after the integer were within-subject factors. The LED timing factor was not included for arithmetic performance, catecholamine, or blood pressure data, which were not collected at these time points. The physiological data collected for each heart beat were analyzed with an added factor of sequential heart beat, with nine levels corresponding to 5 beats preceding the beat when the integer occurred and running to 4 beats following the integer's occurrence. In addition, analyses of variance examined whether physiological changes between the pretask baseline measures and the task measures were statistically significant. For these analyses, baseline data, task-equal probability data, and task-biased probability data formed a factor, termed condition, that had three levels.

The influence of differences in self-report indices and obtained levels of catecholamines was examined by dividing the sample at the median of the index and forming above and below the median groups. For analyses relating arousal and performance, the sample was divided into thirds so that any inverted- $U$ relation could be detected. The basic analyses were then performed with these groups as an added between-subjects factor. A rejection region of $p<.05$ was adopted, with degrees of freedom reduced by the Huynh and Feldt correction for the repeated-measures factors (Winer, 1971).

\section{RESULTS}

\section{Volunteer Characteristics and Baseline Arousal}

Table 1 presents descriptive results comparing the two age gruops. The volunteers were similar in weight, height, and heart rate. The ratio of preejection period to leftventricular ejection time (LVET) was higher in the young, reflecting the increase in contraction duration with age (Lakatta, 1985). The vascular measures (blood pressure, both PTT measures, and PWV) differed between age groups [age main effects, all $\left.F_{\mathbf{s}}(1,24)>5.0\right]$.

Table 1

Volunteer's Physiological Characteristics

\begin{tabular}{lcc}
\hline & \multicolumn{2}{c}{ Age } \\
\cline { 2 - 3 } \multicolumn{1}{c}{ Characteristics } & $\begin{array}{c}\text { Young* } \\
(18-25)\end{array}$ & $\begin{array}{c}\text { Old* } \\
(66-75)\end{array}$ \\
\hline Height (inches) & 70 & 70 \\
Weight (lbs) & 166 & 167 \\
Blood pressure (mmHG systolic/diastolic) & $112 / 70$ & $118 / 73 \dagger$ \\
Heart rate (beats/min) & 61 & 66 \\
PEP/LVET & .44 & $.38 \dagger$ \\
PTT to thumb (msec) & 256 & $222 \dagger$ \\
PTT chest (msec) & 115 & $94 \dagger$ \\
Impedance slope (ohms/sec) & 1.1 & $.8 \dagger$ \\
PWV (m/sec) & 6.6 & $7.5 \dagger$ \\
Epinephrine (pg/ml) & 294 & $610 \dagger$ \\
Norepinephrine (pg/ml) & 371 & $577 \ddagger$ \\
Dopamine (pg/ml) & 552 & 561 \\
\hline
\end{tabular}

$*_{n}=13$. †Age group differences statistically significant (see text). $\ddagger$ Age group difference marginal, $p=.10$. 
Table 2

Volunteers' Self-Reports

\begin{tabular}{|c|c|c|c|}
\hline Self-Report & $\begin{array}{l}\text { Young* } \\
\text { (18-25) }\end{array}$ & $\begin{array}{c}\text { Old }^{*} \\
(66-75)\end{array}$ & \\
\hline Rated stress $(\max =19)$ & 5.8 & $2.5 \dagger$ & \\
\hline Rated arousal $(\max =15)$ & 7.8 & $11.9 \dagger$ & \\
\hline Noise impact $(\min =7, \max =35)$ & 26 & $23 \dagger$ & \\
\hline \multicolumn{4}{|l|}{ Self-report that noise required: } \\
\hline Less focused attention (1 $\ddagger)$ & 3.9 & 3.7 & More (5ł) \\
\hline Less effort & 3.8 & 3.5 & More \\
\hline Less rehearsal & 3.2 & 3.2 & More \\
\hline No vocalizing of integer & 2.9 & 3.2 & Frequent \\
\hline Increased attention to integer & 3.4 & 3.0 & to LEDs \\
\hline
\end{tabular}

${ }^{*} n=13$. †Age difference statistically significant (see text). $\ddagger$ Numerical value of rating corresponding to description. Descriptions on left were rated 1 ; on the right 5 . Mean ratings are presented in the body of the table.

The last three entries of Table 1 show the pretask levels of epinephrine, norepinephrine, and dopamine. The older men had higher levels of plasma epinephrine $[F(1,23)=$ $4.5]$ as well as a trend to higher levels of norepinephrine $[F(1,23)=2.9, p=.10]$. Dopamine did not differ between groups.

Table 2 shows the self-reports of the volunteers. On the SACL, the older group reported greater arousal but less stress during task performance [stress, $F(1,24)=4.6$; arousal, $F(1,24)=6.8]$. The noise-impact reports supported the SACL results: the older volunteers reported less impact from the noise $[F(1,24)=13.1]$. The noise was perceived as increases in effort, focus of attention, and rehearsal, but these perceptions did not differ between age groups.

\section{Performance}

Figure 1 presents mean results for the proportion of key responses that were correct in the two age groups, as a function of the relative probability of LED arrays-biased versus equal-and as a function of LED timing-before or after the integer. Note that the overall probability of any LED's accompanying an integer was .5, and LEDs were less likely overall to occur after the integer $(.3 \times$ $.5=.15)$ than before the integer $(.7 \times .5=.35)$. Responses were slightly faster, but less accurate, for the LEDs after the integers. The mean RT was 539 msec for preinteger LEDs and 503 msec for postinteger LEDs.

The PC results replicate those of the noise condition in the prior experiment of the same design (Jennings et al., 1988b). Performance efficiency on the least probable LEDs (postinteger) was reduced for the young but not the old volunteers. For the younger ones, low-probability LED arrays, whether pre- or postinteger, were associated

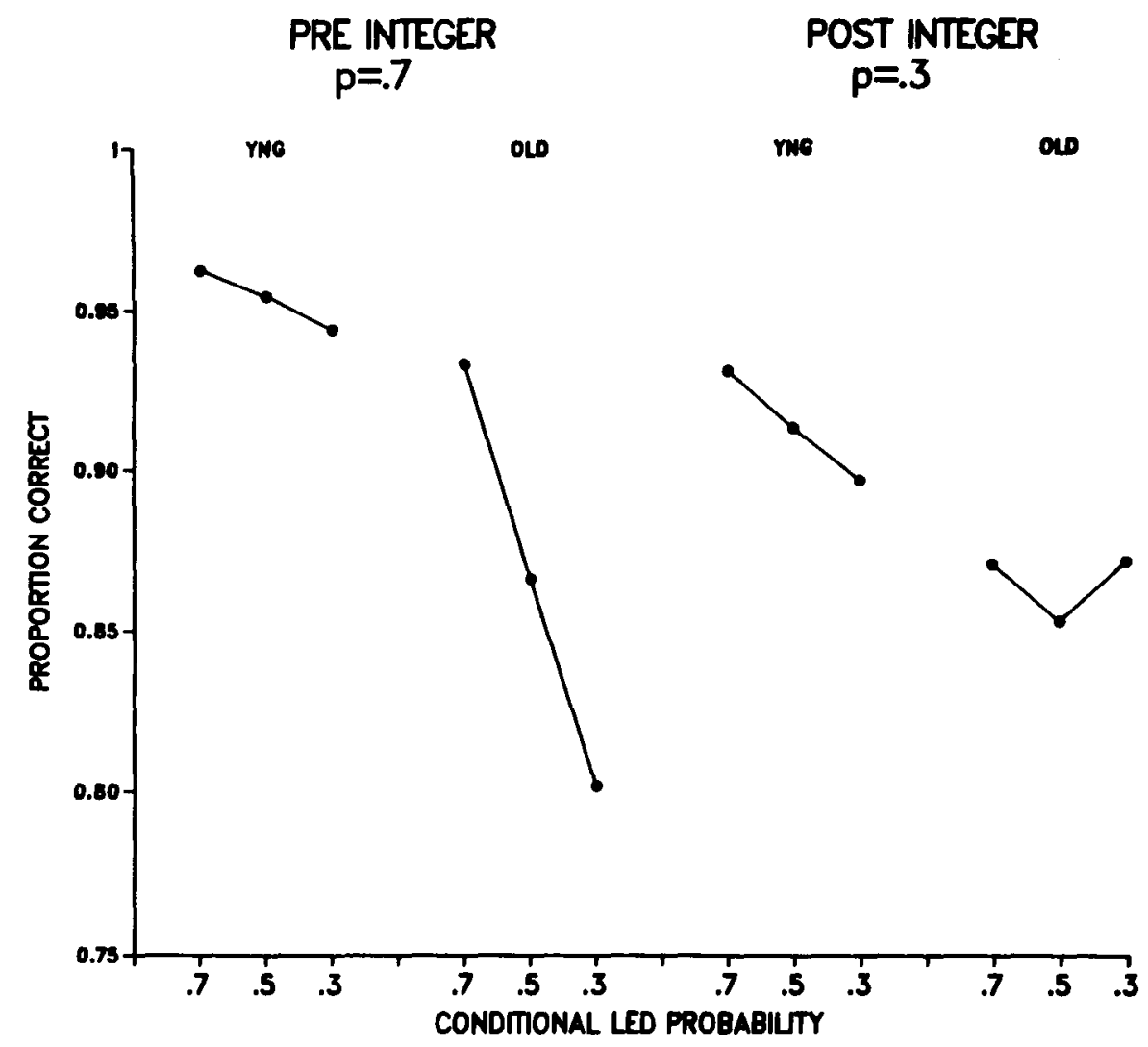

Figure 1. The proportion of correct keypresses during noise for alternative LED diagonal arrays, presented at different probabilities of occurrence. LED stimuli were presented either before (pre) or after (post) the integer presentation for the primary task. If an LED occurred, it was more likely preinteger $(p=.7)$ than postinteger $(p=.3)$. The abscissa represents the relative probability of specific LED arrays, given that an LED occurred. The results for young and old are presented separately. 
with decreased accuracy. For the older ones, however, decreasing probability was associated only with decreasing accuracy for the preinteger LEDs. For the postinteger LEDs, the least and most probable arrays were responded to with equal accuracy, despite the presence of white noise. Statistically, the descriptions are supported by a significant effect of LED timing on $\operatorname{RT}[F(1,24)=16.8]$ and two significant effects on PC: LED timing $[F(1,24)$ $=5.4]$ LED timing $\times$ age $[F(1,24)=4.2]$. The LED timing $\times$ probability $\times$ age factor was not significant, although the direction of the means replicated the results of the prior experiment $[F(2,48)=2.0, p=.15]$.

As instructed, both the young and old volunteers maintained a high level of performance on the arithmetic task ( $99 \%$ correct). The RT showed the typical decrement with age, with an RT of $590 \mathrm{msec}$ for the old and $445 \mathrm{msec}$ for the young, and a PC of .87 for the old and .93 for the young lage effect RT, $F(1,24)=8.9$; PC, $F(1,24)$ $=3.2, p=.08]$. As implied by these results, the older volunteers received the payoff for $\mathrm{RT}$ performance in a smaller proportion of the trials than did the younger volunteers $[.26$ as opposed to $.62 ; F(1,24)=12.0]$.

\section{Catecholamine Responses to the Task}

Since the influence of noise on performance was similar to that found in other experiments (increased bias effects among young but not among old), a next question was whether catecholamine responses indicated an increase in arousal. Over the entire sample, little consistent change between pretask and task periods was observed. None of the relevant $F$ values for base-to-task change approached even marginal significance. Table 3 shows these results and compares them with significant changes observed in the electrophysiological measures.

\section{Cardiovascular Responses to the Task}

Table 3 shows that engagement in the task produced significant increases in heart rate (shown as a decrease in time between beats), diastolic blood pressure, speed of pulse transmission (i.e., a decrease in both PTT measures), and the amplitude of the impedance signal [for con- dition main effect, $F \mathrm{~s}(2,48)>4.8$ ]. Follow-up analyses omitting the baseline data failed to yield any significant effects. This indicates that the base-to-task changes were significant and that there were no significant changes between bias conditions. As in the prior report (Jennings et al., 1988b), neither levels nor responses of the cardiovascular variables were generally related to performance in either a linear or inverted- $U$ relationship. Mean RT and $\mathrm{PC}$, as well as the difference between performance in response to the high- and low-probability stimuli, were examined as a function of the base and change scores for the nine cardiovascular variables. The only significant relations consistent with an inverted- $U$ relationship were a .41 correlation $(d f=24)$ between task-induced change in PWV and the PC difference between high- and lowprobability stimuli.

\section{Event-Related Cardiovascular Responses}

Cardiovascular variables showed brief or phasic responses related to the task events. To the extent that arousal can be equated with reactivity, these responses are relevant with respect to whether or not noise and age induced a consistent arousal response. Table 4 shows the relevant statistical results, which the remainder of the figures will illustrate. Figure 2 shows the IBI responses of the young and old groups to the three LED timing conditions-LED before and after the integer, and no LED occurrence.

Descriptively, the older volunteers responded with less overall cardiac deceleration than the younger volunteers, although the responses of both groups were synchronized with LED timing. A few seconds prior to the anticipated occurrence of the LED/integer event, the IBI started to increase. The occurrence of an LED before the integer led to short-latency accelerative recovery, which was delayed until after the integer for the two other conditions. Accelerative recovery when no LED occurred seemed to be delayed slightly, relative to when the LED did occur after the integer.

Figure 3 uses the same format as Figure 2 to illustrate the PTT results from the thumb measure. The PTT shortened prior to the time of an expected response and then

Table 3

Values of Electrophysiological Indices Showing Significant Tonic Change Compared with Nonsignificant Changes in Catecholamines

\begin{tabular}{lccc}
\hline & \multicolumn{3}{c}{ Experimental Condition } \\
\cline { 2 - 4 } \multicolumn{1}{c}{ Variable } & & \multicolumn{2}{c}{ Noise } \\
\cline { 3 - 4 } & Baseline & Equal Prob. & Bias Prob. \\
\hline IBI (msec) & 946 & 893 & 885 \\
Blood pressure (mmHG systolic/diastolic) & $115 / 71$ & $117 / 74$ & $117 / 74$ \\
PTT-thumb (msec) & 245 & 237 & 236 \\
PTT-chest (msec) & 111 & 103 & 103 \\
Impedance amplitude (ohms) & .64 & .78 & .75 \\
Epinephrine (pg/ml) & 371 & 363 & 369 \\
Norepinephrine (pg/ml) & 422 & 375 & 394 \\
Dopamine (pg/mi) & 565 & 560 & 543 \\
\hline
\end{tabular}

Note-Analyses omitting the baseline fail to show any significant differences. Diastolic, but not systolic, blood pressure showed a significant condition effect. Age did not significantly influence any of the base to task physiological changes. 
Table 4

Significant $F$ Values from Beat-by-Beat Physiological Analyses

\begin{tabular}{lcccccccc}
\hline & \multicolumn{7}{c}{ Term } & \multicolumn{10}{c}{$f^{*}$} & IBI & PTT-TH & SLP-TH & PTT-IMP & SLP-IMP & AMP-IM & PWV \\
\hline nyyyrtbeat & 9,216 & $\mathbf{8 . 8}$ & 5.0 & 5.3 & n.s. & n.s. & n.s. & n.s. \\
Heartbeat $\times$ Age & 9,216 & 3.0 & 3.1 & 2.6 & n.s. & n.s. & 3.0 & $2.4 \dagger$ \\
Heartbeat $\times$ Bias & 9,216 & 2.2 & n.s. & n.s. & n.s. & n.s. & n.s. & n.s. \\
Hearbeat $\times$ LED & 18,432 & 3.5 & n.s. & $2.3 \dagger$ & n.s. & n.s. & $2.2 \dagger$ & n.s. \\
LED & 2,48 & 15.4 & n.s. & n.s. & 3.9 & 3.2 & n.s. & n.s. \\
LED $\times$ Age & 2,48 & n.s. & 3.8 & n.s. & n.s. & n.s. & n.s. & n.s. \\
Bias $\times$ Age & 1,24 & n.s. & n.s. & n.s. & n.s. & 5.5 & n.s. & n.s. \\
\hline
\end{tabular}

*Actual degrees of freedom. For repeated-measures factors, degrees of freedom used to determine significance were adjusted using the Huynh-Feldt technique as reported by the BMDP statistical package (see Winer, 1971). †Not interpreted due to visual evidence of contamination by response artifact.

recovered. As with the IBI, the timing of recovery was manipulated by the LED-timing condition. The thumbPTT response in Figure 3 is of particular interest because of the LED $\times$ age interaction. This reflects the less marked responsivity of the older group to the LED timing.

The LED probability, bias manipulation showed two significant effects on the cardiovascular results-a strong effect on the slope of the impedance signal and a relatively weak effect on the IBI. The slope of the impedance signal was less in the equal-probability condition than in the biased-probability condition for the old but not the young volunteers: young equal $=1.13 \Omega / \mathrm{sec}$, bias $=$ 1.11 ; old equal $=.76 \Omega / \mathrm{sec}$, bias $=.80$. Bias also influenced the timing of anticipatory deceleration of IBI and accelerative recovery. Relative to equal probability, deceleration with equal or greater amplitude was initiated later and terminated earlier in the biased-probability condition. Post hoc tests, however, failed to show significance for specific locations within the IBI sequence.

\section{Catecholamine Levels As Predictors of Performance and Cardiovascular Levels}

Two central questions are whether or not the pretask levels of catecholamine arousal correlated with the levels of cardiovascular arousal indices, and whether or not they predicted subsequent performance more critically. Linear relations were examined for the interrelation of endocrine, cardiovascular, and self-report indices of arousal; both
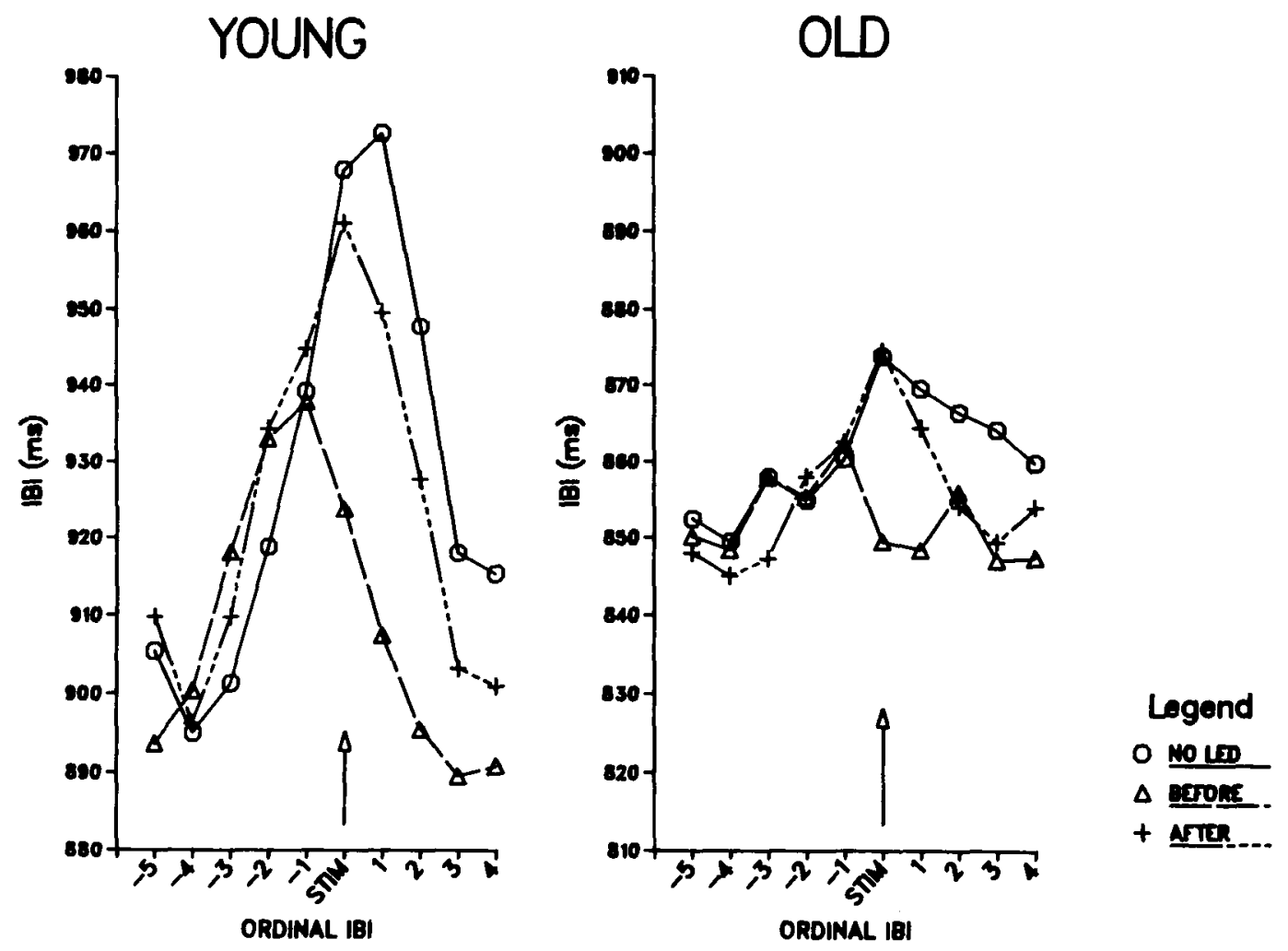

Figure 2. Beat-by-beat values of IBI as a function of whether an LED array occurred either before or after the integer or not at all. The occurrence of the integer, marked by the arrow and "stim" on the abscissa, indicates the IBI during which the integer occurred. Age groups are plotted separately. 

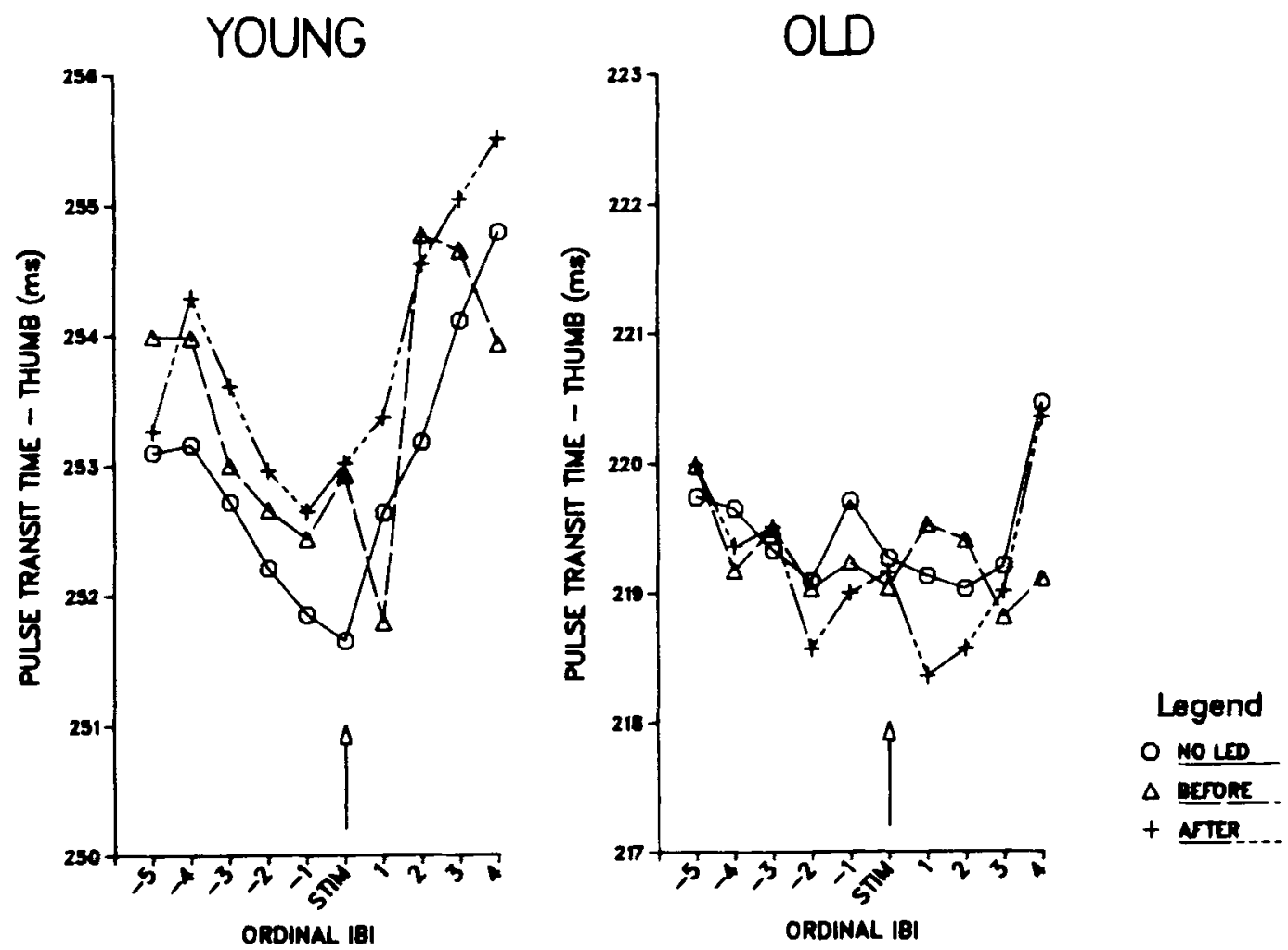

Figure 3. Beat-by-beat values of PTT to the thumb. The occurrence of the integer, marked by the arrow and "stim" on the abscissa, indicates the IBI during which the integer occurred. Age groups are plotted separately.

linear analyses and nonlinear analyses (by grouping in thirds) were used to identify possible inverted-U relations between catecholamines and performance. Both mean levels of performance and scores on the relative performance on low salience stimuli (high- minus lowprobability LED difference) were examined. These analyses were done across age groups, since arousal is hypothesized to be the causal factor with respect to age effects. It should be noted that pretask and task levels of both epinephrine $(r=.71)$ and norepinephrine $(r=.69)$ were highly correlated, while those of epinephrine and norepinephrine were intercorrelated $[r(24)=.58]$. Analyses were done on logarithmically transformed scores in order to normalize the statistical distribution of the catecholamine values. Analyses of catecholamine change were performed using both baseline corrected (adjusted by subtracting linear regression with baseline) and uncorrected scores.

With respect to epinephrine, neither performance index was related in any way to epinephrine levels. Selfreports of stress and noise impact were similarly unrelated, but epinephrine levels were correlated with the degree of arousal assessed by the SACL $[r(24)=.51]$. Separate analyses controlling for age did not eliminate this relation. The degree of focusing experienced due to noise was also correlated (.44) with baseline epinephrine levels. With respect to the cardiovascular measures, epinephrine levels were correlated with levels of the vascular variables. For example, task levels of epinephrine were correlated -.46 with PTT-thumb, -.42 with impedance amplitude, and .51 with PWV.

Like epinephrine, norepinephrine was not significantly related to performance. In fact, the only significant correlation for norepinephrine levels occurred with basal diastolic blood pressure $(r=.47)$, and task-induced change in diastolic pressure $[r(23)=-.42, p<.05]$.

\section{Catecholamine Responses As Predictors of Performance and Cardiovascular Response}

The reactivity of catecholamines to noise or as one aspect of aging might be the means by which arousal is related to performance. Thus, the analyses done for catecholamine levels were repeated for task minus pretask differences in catecholamine.

Epinephrine changes showed an inverted-U relationship with mean RT performance, but were unrelated to selfreport or physiological indices. The division of epinephrine changes yielded the following groups: negative change, $M=-242 \mathrm{pg} / \mathrm{ml}, n=8$, mean age $=49$; little change, $M=33 \mathrm{pg} / \mathrm{ml}, n=8$, mean age $=34$; high change, $M=235 \mathrm{pg} / \mathrm{ml}, n=9$, mean age $=48$. Mean RT (during the equal-probability condition) for the three groups was 604,408 , and $542 \mathrm{msec}[F(2,22)=$ 5.9]. Using age as a covariate in this analysis did not alter 
the result substantially $[F(2,21)=4.4]$, although the result was not significant when the epinephrine groupings were formed from baseline corrected scores. Indices of the salience effect and PC were unrelated to epinephrinechange groups.

Norepinephrine responses were unrelated to performance, but median split analysis suggested two relationships. First, in the older volunteers, a relatively greater norepinephrine response was associated with greater reported stress (SACL $=3.9$ vs. 1.4). In contrast, the younger volunteers with a greater norepinephrine response showed less reported stress (2.5 vs. 8.7) [age $x$ norepinephrine interaction for reported stress in noise, $F(1,21)=8.3$ ]. Second, PWV differed according to age and pretask levels of norepinephrine. With relatively low levels, the young showed a PWV of $6.3 \mathrm{~m} / \mathrm{sec}$, which was higher for the high-norepinephrine group $(7.2 \mathrm{~m} / \mathrm{sec})$. For the old, comparable values were $8.1 \mathrm{~m} / \mathrm{sec}$ for the lownorepinephrine group and 7.1 for the high group [age $\times$ norepinephrine group, $F(1,21)=9.0$ ]. Age differences in PWV were absent when norepinephrine values were relatively high, but the older volunteers showed higher PWV than the younger ones with relatively low norepinephrine levels. Thus, norepinephrine increases were related to self-report of stress and PWV, but differently for the young and old.

\section{DISCUSSION}

Our main question was whether age and noise-induced differences in performance were both due to arousal as defined by catecholamine levels and responses. Ideally, noise and age should change performance efficiency and self-reports. Neuroendocrine and electrophysiological levels and responses would then be examined to see if they mediated the performance changes. Arousal and performance can be defined in various ways within the context of the hypothesis that both under- and overarousal impair performance-that is, according to an inverted-U relationship. We shall examine specifications of the inverted-U relationship that involve both the levels and the response of catecholamines.

\section{Noise, Stress, and Performance}

Loud noise, which typically is viewed as a stressor, in many studies is presumed to induce physiological arousal (Jennings, 1986). Changes in performance are thereby attributed to the presumed arousal. Our earlier study (Jennings et al., 1988b) showed that noise did induce selfreports of mild stress and did alter performance, but that electrophysiological arousal did not occur. Noise produced both the performance of errors and the slowing of responses to low-probability reaction stimuli (biased, postinteger LEDs) among young volunteers, replicating earlier work (e.g., Hockey, 1973). Older volunteers slowed but became more accurate. The present study exactly replicated the noise portion of the earlier study, and thus we expected the same pattern of performance results and self-reports. Although statistically less striking, the same results were obtained. Interpretively, these results for the young are consistent with Broadbent's (1971) and Hockey's (1973) views that peripheral, low-salience events receive less attention in noise than in quiet. We (Jennings et al., 1988b) previously suggested that the old were not adversely influenced by the noise because they were less likely than the young to actively prepare for lowprobability events.

\section{Age and Pretask Arousal}

Arousal, and its relation to performance, can be specified in many ways. In a single study one can test only a small number of these specifications. In the present study, for example, we did not test the within-subject specification of arousal that suggests each individual has his own optimum arousal for tasks of varying complexity (see Gale \& Edwards, 1986). The first specification that we tested was whether the state of high arousal prior to the task (due to age or as an individual difference) explained performance on the task (either overall performance or the effect of noise on performance in response to the low-probability stimuli).

On indices related to heightened arousal, older volunteers appear to differ from younger volunteers consistently. As reviewed by Woodruff (1985), Eisdorfer and his colleagues first noted in a number of studies that older volunteers showed heightened neuroendocrine arousal before, during, and after involvement in challenging tasks. Our results are consistent with altered levels of selfreported arousal, catecholamines, and cardiovascular indices in older volunteers. In three studies (Jennings et al., 1988a, 1988b, and the present one), we have now found that on the SACL older volunteers report higher arousal than do younger volunteers, and that they have vascular indices consistent with sympathetic arousal. The current results add heightened epinephrine levels and a trend toward heightened norepinephrine values in the elderly. The vascular and endocrine results are consistent with the literature (e.g., Lakatta, 1985), although we are unaware of any other consistent reports of heightened self-reports of arousal in the elderly.

Two issues raised by the results are whether the elderly should be classified as overaroused, and if so, whether this is an explanation for their performance. First, the apparent overarousal of the elderly is characteristic only for their pretask levels-physiological changes were generally dampened rather than enlarged in the elderly. Second, our physiological results do not necessarily indicate an activated nervous system that is inducing sympatheticadrenomedullary effects. The vascular results are consistent with known changes that occur with aging in the blood vessels, such as wall-stiffening and atherosclerosis (Lakatta, 1985). The endocrine results also do not directly reflect neural activity (Floras et al., 1986); and factors such as age-related decreases in receptor sensitivity and clearance rates have previously been implicated in the obseved plasma increases with age (Wilkie et al., 1985). 
These nonneural factors may play a role in the current PWV finding. When norepinephrine pretask values were low, the age-induced wall-stiffness effects were present: younger hearts and arteries showed less PWV than did older ones. When norepinephrine was high, the stimulating effect of norepinephrine and an age difference in efficacy was evident: the PWV of young arteries increased more than old arteries, and age differences were attenuated. The interplay of neuropsychological, endocrine, and age-induced structural change suggests that a global concept such as general arousal will not be a useful explanatory concept for the psychophysiology of aging.

Similarly, if a heightened arousal is assumed, performance in the elderly was not predictable by this state. Neither overall performance nor noise-induced selectivity in performance was related to pretask arousal levels. The old in this study and in our prior study (Jennings et al., 1988b) were slow and accurate on low-probability aspects of the dual task under noise conditions, as opposed to the performance of the young. If anything, this result is consistent with an underarousal rather than an overarousal in the elderly. In general, our results question the specification of arousal theory that suggests that arousal levels prior to task performance constrain performance according to an inverted- $U$ function.

\section{Physiological Response to Performance in Noise}

According to a second specification of arousal theory, the effect of a stressor on performance should be mediated through changes in physiological arousal (Eysenck, 1977). Jennings et al. (1988b) observed pretask to task changes in electrophysiological variables, but little change due specifically to performance in noise relative to performance in quiet. Electrophysiological changes, like levels, failed to explain the performance results in their results and in our current ones. For reasons of experimental economy, the quiet condition was not rerun in the current study. Given that the performance and cardiovascular results were replicated, however, we can ask our main question of whether catecholamine arousal can explain the performance results. Replicating the earlier results, both cardiac and vascular indices increased during the task relative to pretask values. Task engagement in noise could be said to produce an electrophysiological arousal. In contrast, catecholamines were not significantly altered. These results, like many before them (e.g., Lacey, 1967), suggest that during relatively typical, moderately stressful, or engaging tasks, the autonomic nervous system and the neuroendocrine system respond differentially rather than en masse, as an undifferentiated arousal system.

Our results are largely consistent with the literature. Catecholamine levels tend to show modest and somewhat variable correlations with cardiovascular variables (e.g., Cohen \& Shmavonian, 1967; Frankenhaeuser \& Lundberg, 1977; Glass et al., 1980; Halter, Stratton, \& Pfeifer, 1984). Given the absence of significant task-induced changes, our data do not help one decide whether young and old differ in catecholamine responsivity. The failure of noise per se to alter catecholamines has been previously reported (see Jennings, 1986), but noise-task combinations with difficult tasks have been previously found to alter catecholamines (Frankenhauser \& Lundberg, 1977). A second difficulty must also be recognized. The pretask levels of catecholamines were relatively high in the present study, which suggests that true resting values may not have been obtained (see, e.g., Wilkie et al., 1985). Conventionally, 20 to $30 \mathrm{~min}$ of maintained repose after catheterization are used to achieve a relatively stable baseline prior to sampling. We did this, but some evidence now suggests that longer periods may be required (Cutler \& Hodes, 1983). We doubt that the high levels are due to any general reactivity to our procedures, because the volunteers had been through them a minimum of three previous times and the three samples drawn in the current experiment did not show a simple decline over time. The levels may reflect an anticipation of challenging tasks, or they may suggest an assay difference between laboratories. If the first possibility is true, the current results may not generalize to catecholamine reactivity based on truly quiescent baselines. Our results do, however, question the specification of arousal theory that suggests that arousal acts through a stressor to elicit a generalized change in autonomic and neuroendocrine response.

\section{Individual Differences in Catecholamine Reactivity and Performance}

A related specification of arousal theory suggest that individual differences in response to a stressor will determine performance according to an inverted-U function. This specification was supported for epinephrine changes. The mean RT on the trials when the probabilities were unbiased was related by means of an inverted- $U$ function to epinephrine change. Interestingly, pretask epinephrine was correlated to self-reports of degree of arousal and focus induced by noise. Thus, the level of beta-adrenergic activation may influence self-perceptions of excitation and involvement, while the degree of task-induced epinephrine change from these levels is related by an inverted-U function to mean performance.

The positive findings should be placed in the context of the negative results. Norepinephrine changes were not related to performance; and even epinephrine changes were not related to the presumed arousing effect of noise on performance: the difference in performance with respect to high- and low-probability stimuli. Of equal importance, epinephrine change was unrelated to caradiovascular change, which calls into question any general physiological arousal. This suggests to us that the noisetask combination specifically influences a beta-adrenergic system related both to self-perceptions of arousal and to general motivational involvement in a task. The limited form of the relationship and the absence of a relationship in cardiovascular measures suggests that general arousal is not a useful explanation for the finding. Others, such as Gale and Edwards (1986), have made similar distinctions among the specifications of arousal theory and would 
argue that for the utility of amending arousal theory to account for results such as the current ones.

\section{A Cognitive-Energetic Alternative}

Stress may not be the sole psychological influence on autonomic and endocrine responses. Normal information processing that produces day-to-day behavior may require the coordination of energetic support provided by both the autonomic and endocrine systems. Stress may then only potentiate the support required by processes necessary for coping effectively with any challenge. Similarly, by changing both information-processing resources and the physiological substrate, aging may alter how autonomic and endocrine support is provided to ongoing processes. This cognitive-energetic view has developed further in a recent volume by Hockey, Gaillard, and Coles (1986), but can be illustrated briefly using the current results.

First, the cardiovascular variables were related to second-by-second changes in the requirements of the task-IBI slowed in anticipation of the LED arrays and the integers; transit times lengthened; and the amplitude, velocity, and slope of the vascular signals tended to increase. Furthermore, age is associated with a drop in amplitude of the changes and a decrease in the degree to which they are synchronized with the task (see Harkins, Moss, Thompson, \& Nowlin, 1976; Jennings et al., 1988a, 1988b; Thompson \& Nowlin, 1973). Second, in a prior report (Jennings et al., 1988b), bias combined with noise altered both vascular response and performance. We suggested earlier that bias may permit the young to prepare a specific response actively-a preparation reflected in heightened peripheral vascular (alpha sympathetic) responses. The present results were based on different measures, but showed an effect on the slope of the impedance signal. On the basis of this measure's correlation with PWV and our earlier pharmacological observations (Jennings \& Choi, 1983a), the impedance-slope difference may reflect alpha-adrenergic innervation as well as betasympathetic activity. In any case, the old and the young both seem to perform differently and to differ in their vascular preparation for low-probability items in noise.

Overall, the associations among (1) temporal anticipation and a concerted cardiac-vascular response, (2) the preparation for a specific response and vascular activation, and (3) the variation in these relationships according to age suggest that the processing induced by a task and the conditions of performance cause specific patterns of cardiovascular support. With aging, this coupling between peripheral support and central processes may weaken. Further work is needed to establish those processes which induce autonomic and endocrine change and determine whether these processes, the peripheral changes, or the coupling is most altered by the aging process. The current results suggest that further work relying solely on the concept of general arousal and a relatively nonspecified inverted- $U$ relationship with performance may not be productive.

\section{REFERENCES}

Broadbent, D. E. (1971). Decision and stress, New York: Academic Press.

Cohen, S. I., Shmavonian, B. M. (1967). Catecholamines, vasomotor conditioning and aging. In L. Gitman (Ed.), Endocrines and aging (pp. 102-141). Springfield, IL: Thomas.

Cutler, N. R., Hodes, J. E. (1983). Assessing the noradrenergic system in normal aging: A review of methodology. Experimental Aging Research, 9, 123-128.

Easterbrook, J. A. (1959). The effect of emotion on cue utilization and the organization of behavior. Psychological Review, 66, 183-201.

EISDORFER, C. (1968). Arousal and performance: Experiments in verbal learning and a tentative theory. In G. Talland (Ed.), Human be havior and aging: Recent advances in research and theory (pp. 189 216). New York: Academic Press.

EYSENCK, M. W. (1977). Attention and arousal. Berlin: Springer-Verlag.

Fleg, J. L., Tzankoff, S. P.. \& Lakatta, E. G. (1985). Age-related augmentation of plasma catecholamines during dynamic exercise in healthy males. Joumal of Gerontology, 59, 1033-1039.

Floras, J., Jones, J. V. Hassan, M. O., Osikowska, B. A., Sever, P. S., \& S1Eight, P. (1986). Failure of plasma norepinephrine to consistently reflect sympathetic activity in humans. Hypertension, $\mathbf{8}$, 641-649.

Frankenhaeuser, M., Lundaerg, U. (1977). The influence of cognitive set on performance and arousal under different noise levels. Motivation \& Emotion, 1. 139-149.

Gale, A., Eowards, J. A. (1986). Individual differences. In M. G. H Coles, E. Donchin, \& S. W. Porges (Eds.), Psychophysiology: Systems, processes, and applicarions (pp. 431-507). New York: Guilford Press

Glass, D. C., Krakoff, L. R., Contrada, R., Hilton, W. F., Kehoe, K., Mannucci, E. G., Collins, C., Snow, B., \& Elting, E. (1980). Effect of harassment and competition upon cardiovascular and plasma catecholamine responses in Type $A$ and Type $B$ individuals. Psychophysiology, 17, 453-463.

Goldberg, P. B., Kreider, M. S., Roberts, J. (1984). Effects of age on the adrenergic cardiac neuroeffector junction. Life Sciences, $35,2585-2591$.

GotTSDANKER, R. (1980). Aging and the maintaining of preparation. Experimental Aging Research, 6, 13-27.

Halter, J. B., Stratton, J. R., Pfeifer, M. A. (1984). Plasma catecholamines and hemodynamic responses to stress states in man. Acta Physiologica Scandinavica, Suppl. 567, 31-38.

Harkins, S. W., Moss, S. F., Thompson, L. W., Nowlin, J. B. (1976). Relationship between central and autonomic nervous system activity: Correlates of psychomotor performance in elderly men. Experimental Aging Research, 2, 409-423.

HoCkey, G. R. J. (1973). Changes in information-selection pattems in multisource monitoring as a function of induced arousal shifts. Journal of Experimental Psychology, 101, 35-42.

HoCkEY, G. R. J. (Ed.) (1983). Stress and fatigue in human performance. Chichester: Wiley.

Hockey, R., Gaillard, A., Coles, M. (1986). Energetics and human information processing. Dordrecht, The Netherlands: Martinus Nijhoff.

JENNINGS, J. R. (1986). Bodily changes during attending. In M. G. H. Coles, E. Donchin, \& S. W. Porges (Eds.), Psychophysiology: Systems, processes and applicarion (pp. 268-289). New York: Guilford Press.

Jennings, J. R., Brock, K., Nebes, R. (1988a). Aging influences upon autonomic response in a divided attention lask. Manuscript submitted for publication.

JenNings, J. R., Brock, K., \& NeBES, R. (1988b). Arousal, attention and aging: Effects of noise and event bias. Manuscript submitted for publication.

Jennings, J. R., Chol, S. (1983a). An arterial to peripheral pulse wave velocity measure. Psychophysiology, 20, 410-418.

Jennings, J. R., Choi, S. (1983b). Use of an optical sensory for arterial to peripheral pulse wave velocity measures. Psychophysiology, 20, 410-418. 
Jennings, J. R., Tahmoush, A. J., \& Redmond, D. (1980). Noninvasive measurement of peripheral vascular activity. In I. Martin \& P. H. Venables (Eds.), Techniques in psychophysiology (pp. 69-137). Chichester, England: Wiley.

LACEY, J. I. (1967). Somatic response patterning and stress: Some revisions of activation theory. In M. H. Appley \& R. Trumbull (Eds.), Psychological stress: Issues in research (pp. 14-42). New York: Appleton-Century-Crofts.

LAKATTA, E. G. (1985). Heart and circulaton. In C. E. Finch, E. L. Schneider, R. C. Adelman, G. M. Martin, \& E. J. Masoro (Eds.), Handbook of the biology of aging (pp. 377-412). New York: Van Nostrand.

MackaY, C. J. (1980). The measurement of mood and psychophysiological activity using self-report techniques. In I. Martin \& P. H. Venables (Eds.), Techniques in psychophysiology (pp. 501-564). Chichester, England: Wiley.

Marsh, G. R., \& Thompson, L. W. (1977). Psychophysiology of aging. In J. E. Birren \& K. W. Schaie (Eds.), Handbook of the psychology of aging (pp. 219-248). New York: Van Nostrand.

Passon, P. G., Peuler, J. D. (1973). A simplified radiometric assay for plasma norepinephrine and epinephrine. Analytical Biochemistry, 51, 618-631.

Sachs, C., Hamberger, B., \&aijser, L. (1985). Cardiovascular responses and plasma catecholamines in old age. Clinical Physiology, 5, 553-565.

Thompson, L. W., Nowun, J. B. (1973). Relation of increased attention to central and autonomic nervous system states. In L. Jarvik, C. Eisdorfer, \& J. Blum (Eds.), Intellectual functioning in adults: Psychological and biological influences (pp. 107-124). New York: Springer.

Wilkie, F. L., Halter, J. B., Prinz, P. N., Benedetti, C., EisDorfer, C., Atwood, B., Yamasakı, D. (1985). Age-related changes in venous catecholamines basally and during epinephrine infusion in man. Journal of Gerontology, 40, 133-140.

WINER, B. J. (1971). Statistical principles in experimental design. New York: McGraw-Hill.

WoODRuFF, D. S. (1985). Arousal, sleep, and aging. In J. E. Birren, K. W. Schaie, V. Bengtson, L. Jarvik, \& T. Salthouse (Eds.), Handbook of the psychology of aging (pp. 261-295). New York: Van Nostrand Reinhold.

Ziegler, M. G., LAKE, C. R., \& Kopin, I. J. (1976). Plasma noradrenaline increases with age. Nature, 261, 333-335.

(Manuscript received December 3, 1987; revision accepted for publication May 7,1988 .) 